The authors state that an estimated GFR $<60 \mathrm{ml} / \mathrm{min} / 1.73 \mathrm{~m}^{2}$ remains the appropriate definition of CKD in the elderly. They suggest, however, that serum cystatin $\mathrm{C}$ levels $\geq 1 \mathrm{mg} / \mathrm{l}$ indicate preclinical kidney disease, and propose that cystatin $\mathrm{C}$ could be a useful biomarker of kidney function, cardiovascular disease, and risk of death in the elderly.

Original article Shlipak MG et al. (2006) Cystatin C and prognosis for cardiovascular and kidney outcomes in elderly persons without chronic kidney disease. Ann Intern Med 145: 237-246

\section{The cancer drug imatinib can cause cardiotoxicity}

Imatinib mesylate is a tyrosine kinase inhibitor used in the treatment of cancers including chronic myeloid leukemia. In their study, however, Kerkelä et al. report that this drug can be cardiotoxic, causing severe left ventricular dysfunction and heart failure in occasional patients.

The authors studied 10 individuals who, having had no previous history of left ventricular dysfunction, presented after a mean $7.2 \pm 5.4$ months of imatinib treatment with major volume overload, symptoms of NYHA class 3-4 heart failure, and low ejection fraction associated with mild left ventricular dilation, among other signs of heart dysfunction. Transmission electron micrographs of myocardial biopsies from two of these individuals and three imatinib-treated mice demonstrated membrane whorls in vacuoles and the sarcoplasmic reticulum, and mitochondrial abnormalities. Ex vivo investigations of mitochondrial function and studies of isolated cardiomyocytes suggested that mitochondria were a primary target of imatinib; mitochondrial dysfunction and the consequent reduction in cellular energetics are intrinsic to imatinib-induced cardiotoxicity. The authors showed that the cardiotoxicity is probably due to inhibition of one specific target of imatinib, the c-Abl protein kinase. Cardiomyocyte death was largely prevented by deactivation of the endoplasmic reticulum stress response induced by imatinib.

The authors recommend further investigations into imatinib-induced cardiotoxicity to define its frequency. In the meantime, patients on imatinib should be watched for heart problems.
Other drugs currently being developed to target c-Abl as well as some other nonreceptor tyrosine kinases could also have some cardiotoxicity, and the authors suggest that the effect of some of these agents on left ventricular function might be assessed prospectively in clinical trials.

Original article Kerkelä R et al. (2006) Cardiotoxicity of the cancer therapeutic agent imatinib mesylate. Nat Med 12: $908-916$

\section{A biomarker for risk assessment in patients with acute coronary syndromes}

Biomarkers are of considerable value for the identification of patients at risk of adverse outcomes in the setting of acute coronary syndromes (ACS). Heart-type fatty acid binding protein $(\mathrm{H}-\mathrm{FABP})$ is rapidly released into the circulation following myocardial injury, but its use as a marker in patients with ACS has not been validated. O'Donoghue et al. have assessed the prognostic utility of H-FABP and conclude that it is associated with poor outcomes and could, therefore, be of use in the risk stratification of patients with ACS.

This study focused on 2,287 patients with ACS who had participated in the doubleblind, randomized, multicenter OPUS-TIMI 16 trial, and for whom baseline blood specimens were available. Elevated $(>8 \mathrm{ng} / \mathrm{ml}$; the lowest detectable threshold) H-FABP concentration was identified in 332 (14.5\%) patients and was a significant predictor of major adverse clinical events over the subsequent 10 -month period when compared with undetectable $(\leq 8 \mathrm{ng} / \mathrm{ml})$ H-FABP levels $(23.5 \%$ vs $9.3 \%$; hazard ratio $2.6,95 \% \mathrm{Cl} 1.9-3.5 ; P<0.001)$.

High H-FABP concentration was associated with risk of death and major cardiac events, and was independent of troponin-I and brain natriuretic peptide. The authors suggest that $\mathrm{H}-\mathrm{FABP}$ assays could be used as part of a multimarker approach to risk assessment for patients with ACS, although they recommend confirming the utility of the protein in a more heterogeneous patient sample, and also in the context of a new, more-sensitive troponin-I assay.

Original article O’Donoghue M et al. (2006) Prognostic utility of heart-type fatty acid binding protein in patients with acute coronary syndromes. Circulation 114: 550-557 